\title{
Subcellular localization of Mitf in monocytic cells
}

\author{
Ssu-Yi Lu $\cdot$ Hsiao-Ching Wan $\cdot$ Mengtao Li $\cdot$ \\ Yi-Ling Lin
}

Accepted: 15 April 2010 / Published online: 1 May 2010

(C) The Author(s) 2010. This article is published with open access at Springerlink.com

\begin{abstract}
Microphthalmia-associated transcription factor (Mitf) is a transcription factor that plays an important role in regulating the development of several cell lineages. The subcellular localization of Mitf is dynamic and is associated with its transcription activity. In this study, we examined factors that affect its subcellular localization in cells derived from the monocytic lineage since Mitf is present abundantly in these cells. We identified a domain encoded by Mitf exon $1 \mathrm{~B} 1 \mathrm{~b}$ to be important for Mitf to commute between the cytoplasm and the nucleus. Deletion of this domain disrupts the shuttling of Mitf to the cytoplasm and results in its retention in the nucleus. M-CSF and RANKL both induce nuclear translocation of Mitf. We showed that Mitf nuclear transport is greatly influenced by ratio of M$\mathrm{CSF} / \mathrm{Mitf}$ protein expression. In addition, cell attachment to a solid surface also is needed for the nuclear transport of Mitf.
\end{abstract}

S.-Y. Lu and H.-C. Wan contributed equally to this work.

S.-Y. Lu • M. Li · Y.-L. Lin

Section of Oral Pathology,

Department of Diagnostic and Surgical Sciences,

School of Dentistry, University of California,

Los Angeles, CA 90095, USA

H.-C. Wan

Department of Medicine, Beth Israel Deaconess Medical Center,

DA-617, 330 Brookline Ave., Boston, MA 02215, USA

Y.-L. Lin $(\varangle)$

CHS 53-058B, 10833 Le Conte Ave.,

Los Angeles, CA 90095, USA

e-mail: ylin@dentistry.ucla.edu
Keywords M-CSF $\cdot$ Monocytic cells $\cdot$ Nuclear localization signal $\cdot$ Attachment $\cdot$ Transcription factor

\section{Introduction}

Genes are tightly regulated in their expression levels, patterns and timings. Throughout evolution, cells have adopted various sophisticated ways to control gene expression. Many of these control mechanisms are aimed at transcription factors. One common regulatory strategy is to sequester transcription factors in the cytoplasm. By doing so, the transcription factors remain inactive in the cells and enter the nucleus to turn on target genes only when cells receive appropriate signals. NF- $\kappa \mathrm{B}$ and NFAT are two well-studied examples of transcription factors capable of commuting between the cytoplasmic and nuclear compartments in response to environmental stimuli. For NF- $\kappa \mathrm{B}$, it is normally sequestered in the cytoplasm by its inhibitor $\mathrm{I} \kappa \mathrm{B}$. Signal transduction leads to $\mathrm{I} \kappa \mathrm{B}$ phosphorylation and subsequent release of NF- $\kappa \mathrm{B}$ to translocate to the nucleus (Mercurio and Manning 1999); for NFAT, signal transduction leads to activation of calcineurin, which results in dephosphorylation and nuclear translocation of NFAT (Hogan et al. 2003).

Microphthalmia-associated transcription factor (Mitf) is a basic helix-loop-helix leucine zipper (bHLH-Zip) transcription factor that is able to form homo- and heterodimers with other MiT family members (Tfeb, Tfec and Tfe3) in vitro (Hemesath et al. 1994). Mitf dimers bind to an E-box consensus sequence $\mathrm{CA}[\mathrm{C} / \mathrm{T}] \mathrm{GTG}$ in the target promoters to activate the genes (Hemesath et al. 1994; Steingrimsson et al. 1994). Mitf gene exhibits a split promoter design, which allows it to generate multiple structurally and biologically distinct proteins (Hershey and Fisher 
2005). This property enables Mitf to perform diverse biological functions. Disruption of Mitf gene in mice inhibits melanocytogenesis and retinal pigmented epithelium development, affects the number and function of mast cells, and inhibits late differentiation stage of osteoclasts (Steingrimsson et al. 2004). Mutations of Mitf also causes dysfunctions of several hematopoietic cells (Rohan et al. 1997; Roundy et al. 1999; Stechschulte et al. 1987; Thesingh and Scherft 1985 ) including macrophages, suggesting regulatory roles of Mitf in these cells.

Mitf contains a nuclear localization signal (NLS), which directs its nuclear translocation upon receiving appropriate signals from the cells. Mitf shows different subcellular localization patterns in different types of cells. For example, in normal and malignant melanocytes, Mitf is predominantly a nuclear protein while in breast tumors, the protein can be predominantly nuclear or cytoplasmic (Granter et al. 2002). Recently, Mitf was shown to be able to shuttle between the cytoplasmic and nuclear compartments in macrophages (Bronisz et al. 2006). These studies suggest that the localization of Mitf is associated with the different growth and differentiation programs present in these cells.

\section{Materials and methods}

Reagents

Anti $(\alpha)$-HA antibody $(\mathrm{Ab})$ was obtained from Boehringer Mannheim, Germany. $\alpha$-Mitf Ab (C5) was obtained from Calbiochem, San Diego, CA and as a gift from Dr. David E. Fisher, Massachusetts General Hospital, Boston, MA. Fluor-conjugated $\alpha$-HA and goat $\alpha$-mouse Abs, DAPI and phalloidin were purchased from Invitrogen, Carlsbad, CA.

Plasmid constructs

Mitf and its deletion mutants were amplified from a previously constructed plasmid containing Mitf isoform A (MitfA) sequence and from mouse cDNA. Two overlapping PCR forward primers were used to generate the Mitf products with a desired restriction enzyme site and a Kozak sequence. The Mitf PCR products were eventually cloned into a retroviral vector pMSCViG (modified from pMSCV, Clontech, Mountain View, CA). pMSCViG has a C-terminal HA-tag. Forward primers used: Mitf-A, 5'-ATGCA GTCCGAATCGGGA-3', 5' -AGATCTACCATGGCGAT GCAGTCCGA-3'; Mitf-dA, 5'-CTACCATGGTGATGAG TTCTGCAGAGCAT-3', 5' -TTGAGATCTACCATGGT GATGAGTTCTGCAG-3'; Mitf-dAB: 5'-CTACCATGG
TGCAGACCCACCTGGAA-3'， 5'-TTAAGATCTACCA TGGTGCAGACCC- $3^{\prime}$. Reverse primer used: for pMSCViG cloning, 5'-TAAGTCGACCACACGCATGCTCCGT TTCT-3'.

\section{Cell cultures}

Mouse bone marrow from 2-4-month-old wild-type mice was harvested by flushing cold MEM $\alpha$ through the femur and tibia with a 22-gauge needle. Human peripheral blood mononuclear cells (hPBMC) were ficoll fractionated and resuspended in serum-free media. Monocytes were subsequently purified by plate adhesion. Both human and mouse cells were cultured in media supplemented with $100 \mathrm{ng} / \mathrm{mL}$ M-CSF or appropriate cytokines as indicated in the figure legends. RAW264.7, MDA-MB231 and 3T3 were obtained from ATCC (Manassas, VA).

Immunofluorescent staining

Cells were fixed in $2 \%$ paraformaldehyde, permeabilized with $0.1 \%$ saponin and blocked with $10 \%$ goat serum. To detect endogenous Mitf signals, cells were incubated with the $\alpha$-Mitf C5 Ab followed by an Alexa fluor-conjugated secondary Ab. To detect Mitf-HA fusion proteins, cells were incubated with an Alexa fluor-conjugated $\alpha-\mathrm{HA} \mathrm{Ab}$. Cells were also counterstained with phalloidin and DAPI, which stain actin and nuclei, respectively.

Retrovirus production and infection

293 FT cells (Invitrogen) were transfected with a retroviral vector together with pVPack-GP (Stratagene, Cedar Creek, Texas) and pVSV-G (Clontech). Media of the transfected cells were collected and used to infect cells.

Western blot

Cells were lysed in a lysis buffer containing 2\% SDS, $50 \mathrm{mM}$ Tris (pH 6.8), $10 \%$ glycerol, $0.1 \%$ benzonase (Sigma-Aldrich), protease inhibitors (Sigma-Aldrich, Sigmafast) and phosphatase inhibitors ( $20 \mathrm{mM}$ sodium pyrophosphate, $10 \mathrm{mM}$ sodium fluoride and $1 \mathrm{mM}$ sodium orthovanadate). The lysates were rotated at room temperature for $15 \mathrm{~min}$, cleared by centrifugation, quantified and separated by SDS-polyacrylamide gels. Proteins were transferred to nitrocellulose membranes. The membrane was blocked and incubated with the appropriate primary $\mathrm{Ab}$ followed by a HRP-conjugated secondary $\mathrm{Ab}$. The protein signal was developed with ECL reagents (Pierce Biotechnology). 


\section{Results}

Mitf cytoplasmic-nuclear shuttling is affected by a domain encoded by exon $1 \mathrm{~B} 1 \mathrm{~b}$

The Mitf gene locus contains at least nine isoform-specific promoters (Amae et al. 1998; Fuse et al. 1999; Hershey and Fisher 2005; Oboki et al. 2002; Shiohara et al. 2009; Steingrimsson et al. 1994; Takeda et al. 2002; Takemoto et al. 2002; Udono et al. 2000). Each Mitf isoform transcript contains an isoform-specific first exon that is spliced to exon $1 \mathrm{~B} 1 \mathrm{~b}$ and then to common exons 2-9, which encode the known functional motifs (Fig. 1). The only exception is Mitf-M. Exon $1 \mathrm{M}$ is located $3^{\prime}$ to exon $1 \mathrm{~B} 1 \mathrm{~b}$; therefore exon $1 \mathrm{M}$ splices directly to exons $2-9$, resulting in a shortened amino terminus in Mitf-M due to the absence of exon $1 \mathrm{~B} 1 \mathrm{~b}$ in its sequence (Fig. 1b). Mitf-M is not present in monocytic cells as its expression is restricted to melanocytes and mast cells derived from certain tissue sources (Oboki et al. 2002). In contrast to the Mitf in monocytic cells (Bronisz et al. 2006), endogenous Mitf-M has not been reported to be cytoplasmic. Since all Mitf isoforms except Mitf-M have exon 1B1b in their sequences, we investigated whether exon $1 \mathrm{~B} 1 \mathrm{~b}$ encodes a domain that allows cytoplasmic shuttling of Mitf protein. Since Mitf-A is an abundant isoform in monocytic cells, Mitf-A and its two deletion mutants, Mitf-dA and Mitf-dAB, were used to examine the subcellular localization of Mitf in monocytic cells. The mutant Mitf-dA refers to Mitf-A with a deletion of isoform-specific domain A; the mutant Mitf-dAB refers to Mitf-A without domain $\mathrm{A}$ and the domain encoded by exon 1B1b (Fig. 2a).

RAW264.7 cells, a mouse monocytic cell line, were infected with retroviruses expressing Mitf-A, Mitf-dA, Mitf-dAB or a null vector as control. The HA-tagged Mitf$\mathrm{A}$ and its mutants were expressed and detected by $\alpha$-HA Ab (Fig. 2a). The immunofluorescent staining of the infected
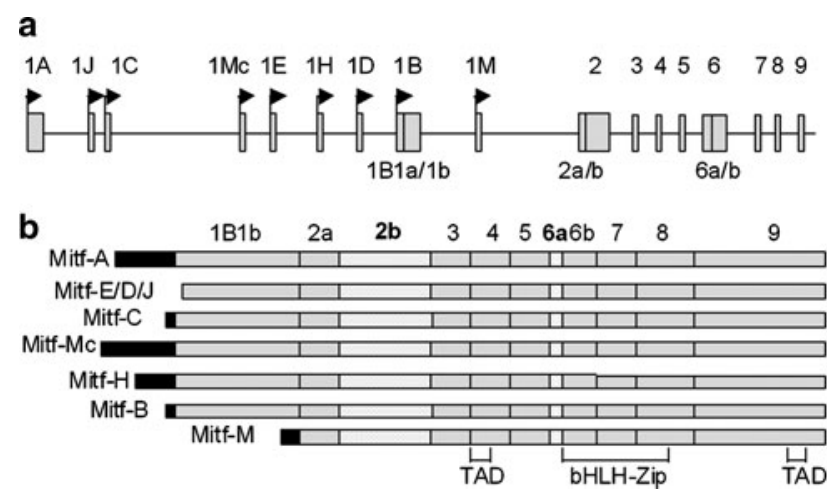

Fig. 1 a Exon organization of the Mitf gene. b Schematic representation of Mitf isoforms. TAD transactivation domain. bHLH-Zip basic helix-loop-helix leucine zipper
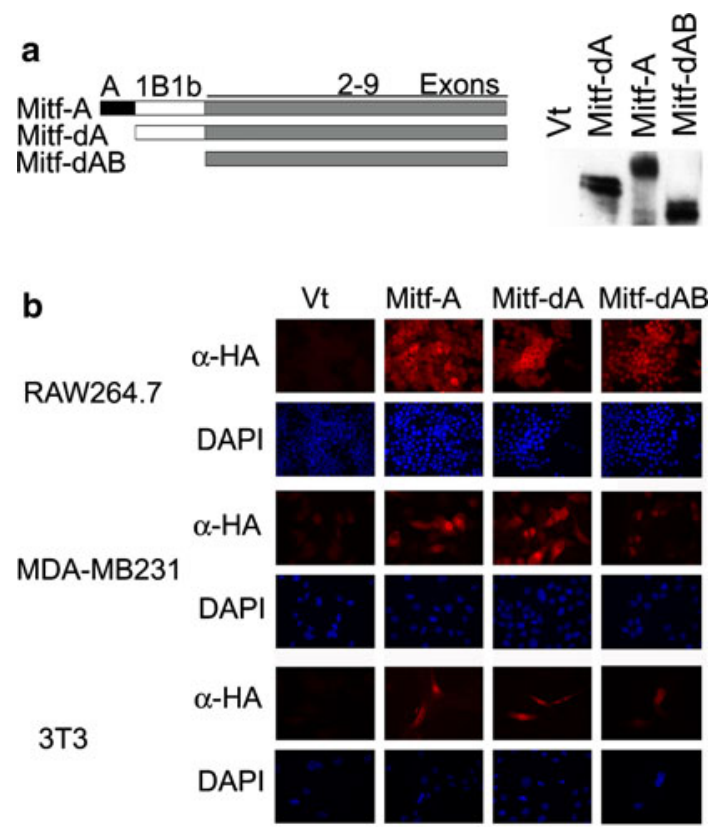

Fig. 2 Mitf exon 1B1b encodes a sequence interfering with Mitf nuclear localization. a Left Schematic representation of Mitf constructs used in the study with exon numbers labeled on the top of the boxed sequences. Right Western blot of recombinant Mitf-HA proteins ectopically expressed in RAW264.7 cells. The recombinant proteins were detected with $\alpha$-HA Ab. b Subcellular localization of recombinant Mitf proteins in various cell lines. Cells were infected with Mitf$\mathrm{HA}$ retroviruses and Mitf-HA expression was detected with $\alpha-\mathrm{HA} \mathrm{Ab}$ by immunofluorescent staining ( $r e d)$. Cells were counterstained with DAPI, which stains the nuclei (blue)

cells showed that Mitf-A and Mitf-dA were present in both cytoplasmic and nuclear compartments, while Mitf-dAB was predominantly nuclear (Fig. 2b). To exclude the possibility that the cell type may affect the protein subcellular localization, similar experiments were also carried out with 3 T3 fibroblast cells and MDA-MB231 breast cancer cells (Fig. 2b). The results obtained from these two non-monocytic cells were comparable with that of RAW264.7 cells. In all cases, deletion of exon A had no noticeable effect on Mitf's ability to shuttle between the nuclear and cytoplasmic compartments, while deletion of exon $1 \mathrm{~B} 1 \mathrm{~b}$ resulted in a Mitf protein that was predominantly nuclear. To ensure that the HA sequence present in the carboxyl termini of the recombinant proteins was not interfering with their subcellular localization, Mitf constructs that have no HA-tag were also used (data not shown). The results were similar, which indicated that the C-terminal HA sequence did not interfere with the subcellular localization of the Mitf proteins. These results suggest that the sequence encoded by exon $1 \mathrm{~B} 1 \mathrm{~b}$ plays an important role in Mitf protein's ability to shuttle to the cytoplasmic compartment. Deletion of this exon from the Mitf sequence permits the NLS to become the dominant factor in determining Mitf's subcellular localization and the 
protein become predominantly nuclear, as in the case of Mitf-dAB and Mitf-M.

Mitf nuclear localization promoted by M-CSF is dose dependent

Although Mitf has been reported as a nuclear protein in melanocytes, in mouse bone marrow macrophages (mBMM), it can shuttle between the cytoplasm and nucleus and its nuclear translocation is promoted by cytokines M-CSF and RANKL (Bronisz et al. 2006). Mitf exhibits nuclear localization when $\mathrm{mBMM}$ are cultured in media containing M-CSF; when M-CSF is withdrawn from the media, Mitf protein is redistributed to the cytoplasmic compartment (Bronisz et al. 2006).

However, when we examined the subcellular localization of Mitf protein in RAW264.7 cells, a monocytic cell line, the protein was present in both nuclear and cytoplasmic compartments, and addition of M-CSF had no effect on promoting nuclear localization of Mitf protein (Fig. 3). Kinetic studies showed that RAW264.7 cells stimulated with M-CSF from $15 \mathrm{~min}$ to several days with media change every two days did not reveal any differences in the subcellular localization of Mitf (data not shown). In contrast, when RAW264.7 cells were treated with RANKL, Mitf started accumulating in the nuclei while the cells differentiated toward osteoclast-like cells (Fig. 3). Although M-CSF had no effect on Mitf's subcellular localization in RAW264.7 cells, treating these cells with M-CSF resulted in cellular proliferation (data not shown), indicating that RAW264.7 cells have functional c-fms, the M-CSF receptor, on the cell surfaces.

Fowles et al. have pointed out that RAW264.7 cells may be defective in c-fms trafficking to the cell surfaces; therefore, while these cells have functional c-fms that can respond to M-CSF, they exhibit a quantitative deficiency of M-CSF signaling (Fowles et al. 2000). Therefore, we hypothesize that nuclear localization of Mitf promoted by M-CSF may be affected by the quantity of M-CSF signal,

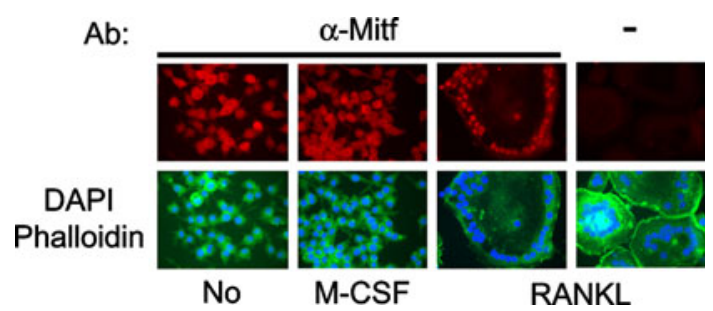

Fig. 3 RANKL, but not M-CSF, induces nuclear accumulation of Mitf protein in RAW264.7 cells. RAW264.7 cells were treated with vehicle, M-CSF (100 ng/mL) or RANKL ( $100 \mathrm{ng} / \mathrm{mL}$ ) for 3 days. Mitf expression was detected by $\alpha$-Mitf Ab by immunofluorescent staining (red). Cells were counterstained with DAPI and phalloidin, which stain nuclei (blue) and actin (green), respectively reflected by the ratio of M-CSF signal to Mitf protein levels. This was investigated by expressing different levels of Mitf protein in mBMM and determined whether Mitf localization would be affected according to the protein levels when the cells were cultured in a fixed amount of M-CSF. We over-expressed Mitf-A, the most abundant isoform, in these primary cells with a recombinant retrovirus. When mBMM were infected with the undiluted retrovirus, immunofluorescent staining with $\alpha$-HA $\mathrm{Ab}$, it was revealed that the recombinant Mitf-A was present predominantly in the cytoplasm with only a small portion of cells exhibiting a nuclear staining of Mitf (Fig. 4). However, when mBMM were infected with the virus diluted by five fold, the recombinant protein detected was predominantly in the nuclei of the infected cells (Fig. 4). We have conducted both Western blot and immunofluorescence studies to confirm that cells infected with diluted viruses had less Mitf expression on a per cell basis and these cells did not have a lower percentage of infection rate. Thus, the experiment demonstrated that a higher titer of Mitf-A retrovirus was associated with an outcome of increased cytoplasmic localization of MitfA. Since the undiluted virus resulted in a higher expression level of Mitf-A protein, the results implied that when M-CSF/Mitf ratio decreased due to increased Mitf expression, there would not be sufficient quantities of M-CSF signal to direct Mitf protein to the nuclei.

Endogenous Mitf was predominantly a nuclear protein in $\mathrm{mBMM}$ when the cells were cultured in $100 \mathrm{ng} / \mathrm{mL}$ M-CSF with regular replenishment of fresh M-CSF and depletion of M-CSF results in Mitf shuttling to the cytoplasm (Bronisz et al. 2006). Since M-CSF activity would gradually decline with time in the culture, we hypothesized that fluctuation of M-CSF activity may affect the subcellular localization of Mitf. To further investigate this phenomenon, mBMM were infected with control and Mitf retroviruses and a time course study was conducted to examine the subcellular localization of Mitf-A, Mitf-dA or Mitf-dAB in these cells following M-CSF stimulation. The results

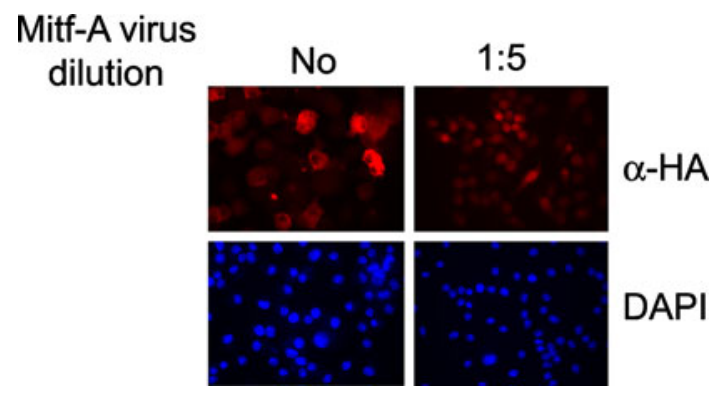

Fig. 4 Expression levels of Mitf-A affect its subcellular localization in primary mouse macrophages. Mouse macrophages were infected with Mitf-A retroviruses with no dilution or five fold dilution. Mitf-HA (red) was detected with $\alpha$-HA Ab by immunofluorescent staining. Cells were counterstained with DAPI, which stains the nuclei (blue) 


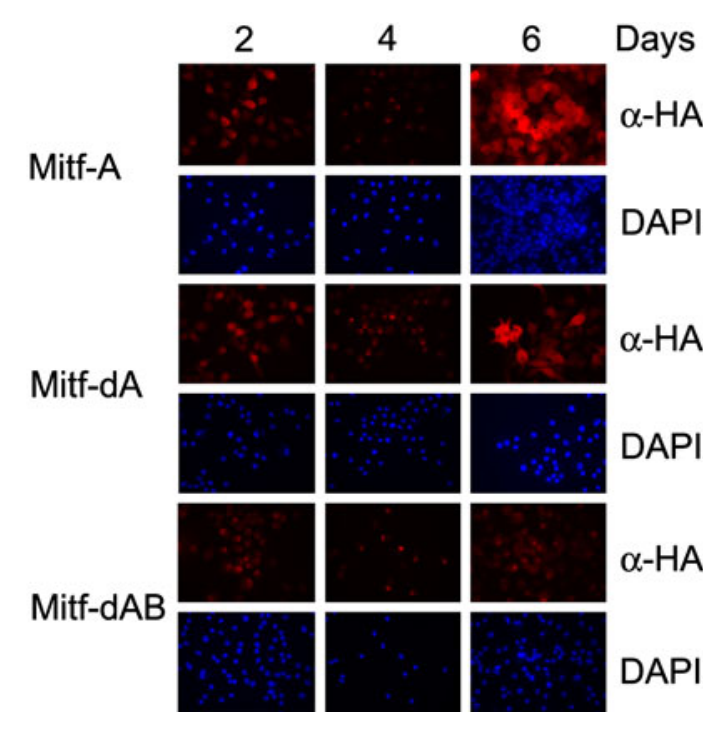

Fig. 5 Subcellular localization of recombinant Mitf proteins in primary macrophages depends on the activity of M-CSF in the media. Mouse macrophages maintained in media containing M-CSF were infected with Mitf retroviruses. Media were replenished with fresh M-CSF on day 3. Mitf-HA (red) was detected with $\alpha$-HA Ab by immunofluorescent staining. Cells were counterstained with DAPI, which stains the nuclei (blue)

showed that while the over-expressed recombinant Mitf-A and Mitf-dA were initially present in both nuclear and cytoplasmic compartments after infection (Fig. 5, day 2), replenishment of media containing fresh M-CSF on day 3 resulted in synchronous nuclear accumulation of Mitf proteins in all infected cells within $24 \mathrm{~h}$, and Mitf becomes predominantly nuclear (Fig. 5, day 4). This was followed by gradual redistribution of the Mitf proteins to both cytoplasmic and nuclear compartments. On day 6, when the protein expression peaked, both proteins became predominantly cytoplasmic (Fig. 5, day 6). As expected, Mitf-dAB, the mutant protein that was defective in cytoplasmic shuttling, remained in the nucleus and was not affected by the decreased M-CSF activity in the media (Fig. 5).

In summary, we found that levels of Mitf and M-CSF influence the Mitf localization. In the presence of sufficient amounts of M-CSF, Mitf protein becomes nuclear. However, if the Mitf protein levels exceed the M-CSF required to keep the protein nuclear, a portion of the Mitf protein shuttles from the nuclear to the cytoplasmic compartment and the protein can be present in both compartments or become predominantly cytoplasmic.

Cell attachment to a solid surface affects the subcellular localization of Mitf in monocytic cells

When cultured on plates, mBMM attached to the surface and assumed a plump, epithelioid shape. Mitf was predominantly nuclear in these cells (Fig. 6a) as long as the cultures were regularly replenished with media containing fresh M-CSF. Similarly, human monocytes cultured in the presence of M-CSF were mostly spindle or epithelioid in shape and the immunofluorescent staining with $\alpha$-Mitf $\mathrm{Ab}$ showed that the Mitf protein was predominantly nuclear (Fig. 6b). However, human monocytes formed colony foci when cultured longer than 2 weeks in the presence of M-CSF. These colonies were composed of a morphologically distinct group of cells that were round and refractile. The colony cells were easily detached from the culture surface and dissociated from each other by gentle physical agitation. Interestingly, the colony cells exhibited a strong cytoplasmic staining of Mitf despite the presence of M-CSF in the media (Fig. 6c), while the tightly attached spindle/epithelioid cells in the same culture exhibited nuclear Mitf staining. Furthermore, Mitf in these colony cells stayed in the cytoplasm even when the cells were treated with both M-CSF and RANKL (data not shown), indicating that the nuclear localization of Mitf in these cells requires signal(s) additional to M-CSF and RANKL stimulation.

We noted that when the colony cells were dissociated from each other by physical force, they attached to the adjacent surface of the culture plate, assumed a spindle/epithelioid shape and exhibited a nuclear staining of Mitf protein. Based on this observation, we subsequently investigated whether cell attachment can affect the subcellular localization of Mitf. The colony cells were first harvested in cloning rings to prevent contamination from other cells in the culture and then subjected to several washes to remove M-CSF before resuspension in PBS. Cytospin was subsequently performed to attach the cells to glass slides with centrifugation force. The attached cells were immediately fixed and Mitf localization was examined by immunofluorescent staining with $\alpha$-Mitf $\mathrm{Ab}$. In contrast to the cytoplasmic localization previously observed, Mitf became predominantly nuclear in these attached cells even in the absence of cytokines during the attachment procedure (Fig. 6d). The results clearly indicate that cell attachment to a solid surface triggers a cytokine-independent signal that is necessary for Mitf nuclear translocation in human monocytic cells.

\section{Discussion}

The intracellular shuttling of Mitf is an important mechanism to regulate its transcription activity during cell differentiation and development. In this study, we identify a domain encoded by Mitf exon 1B1b (domain 1B1b), which appears to play an important role in regulating the cytoplasmic shuttling of Mitf. It should be mentioned that the domain $1 \mathrm{~B} 1 \mathrm{~b}$ does not overlap with the previously defined 


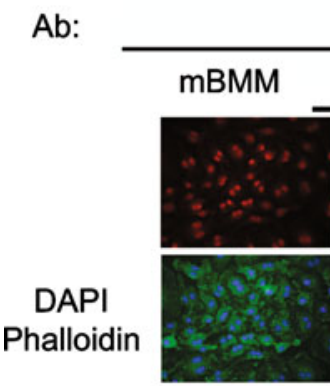

a
$\alpha$-Mitf

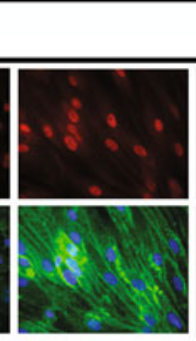

b

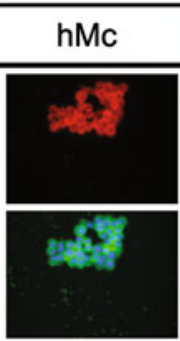

C
Fig. 6 Subcellular localization of Mitf in primary mouse and human macrophages. a Nuclear localization of Mitf in mouse macrophages cultured in media supplemented with $100 \mathrm{ng} / \mathrm{mL}$ M-CSF. b Nuclear localization of Mitf in human macrophages cultured in media supplemented with $100 \mathrm{ng} / \mathrm{mL}$ M-CSF. c Cytoplasmic localization of Mitf in colony cells in human macrophage cultures. These cells have a different morphology from those tightly attached cells shown in b. d Nuclear localization of Mitf in the colony cells after attaching to glass slides by cytospin. Mitf ( $r e d$ ) was detected by $\alpha$-Mitf Ab by immunofluorescent staining. Cells were counterstained with DAPI and phalloidin, which stain nuclei (blue) and actin (green), respectively. $\mathbf{e}$ and $\mathbf{f}$ are immunofluorescent staining control for human and mouse cells staining with only secondary Abs serine 173, phosphorylation of which promotes Mitf interaction with cytoplasmic protein 14-3-3 and interferes with the nuclear localization of Mitf (Bronisz et al. 2006). The domain $1 \mathrm{~B} 1 \mathrm{~b}$ is located near the N-termini of all Mitf isoforms except Mitf-M. According to our results, any Mitf isoform that lacks the domain $1 \mathrm{~B} 1 \mathrm{~b}$ should remain in the nucleus and lead to prolonged gene activation. Indeed, a study showed that Mitf-M exhibits stronger transcription activity than other Mitf isoforms (Takeda et al. 2002). We speculate that the absence of the domain 1B1b in Mitf-M may contribute to its increased transcription activity and the domain 1B1b may mediate cytoplasmic shuttling of Mitf by partially masking the NLS or by interacting with a cytoplasmic-anchoring protein. In our study, the deletion of the domain 1B1b from Mitf-A generated a protein similar to Mitf-M, and this mutant displayed almost $100 \%$ nuclear localization, which also is not affected by M-CSF levels in the media. Interestingly, although endogenous Mitf-M has never been described as cytoplasmic, yet when Mitf-M fuses to an amino GFP, the GFP-Mitf-M protein displays a mixed localization with approximately $60 \%$ in the cytoplasm and $40 \%$ in the nucleus (Bronisz et al. 2006), suggesting that the amino GFP interferes with the nuclear localization of Mitf-M. The observation that GFP-Mitf-M has similar subcellular localization as Mitf-A suggests that an extended amino domain, regardless of its sequence, is able to interfere with the nuclear localization of Mitf-M. Therefore, an intramolecular masking mechanism is favored over the cytoplasmic anchoring mechanism, as the latter requires protein-protein interaction, which is sequence dependent.

$\mathrm{M}-\mathrm{CSF}$ is the primary regulator of the survival, proliferation, differentiation and function of monocytic cells (Chitu and Stanley 2006; Lagasse and Weissman 1997). RANKL is not essential for monocytic cell survival; instead it provides osteoclast differentiation signals for osteoclast precursors derived from monocytic cells (Lacey et al. 1998). Both M-CSF and RANKL pathways lead to activation and phosphorylation of Mitf in macrophages (Mansky et al. 2002; Weilbaecher et al. 2001). However, while both M-CSF and RANKL stimulations result in nuclear localization of Mitf in primary macrophages (Bronisz et al. 2006), only RANKL has effect on Mitf's subcellular localization in RAW264.7 cells, a mouse monocytic cell line. This is most likely due to deficiency of c-fms receptor trafficking, which results in reduced $\mathrm{M}-\mathrm{CSF} / \mathrm{c}-\mathrm{fms}$ signals in RAW264.7 cells (Fowles et al. 2000). Previous work by Bronisz et al. suggested that M-CSF/RANKL combined signals disrupt interaction between Mitf and c-TAK1 in RAW264.7 cells (Bronisz et al. 2006). Consequently, Mitf is released from its cytoplasmic-anchoring protein 14-3-3 and enters the nuclei. While Bronisz et al's data show that Mitf nuclear transport can be regulated by c-TAK1 inhibition induced by combined RANKL/M-CSF treatment, our data demonstrate that RANKL alone is sufficient to induce Mitf translocation in these cells. In addition to M-CSF and RANKL stimulation, signals generated from interactions between the cells and its microenvironment appear to be critical for Mitf's localization.

We propose that Mitf's subcellular localization is regulated by an intramolecular NLS masking mechanism depicted in Fig. 7. In this model, M-CSF signals unmask the NLS, possibly through protein modification, and Mitf is imported to the nucleus; in the absence of M-CSF, the domain 1B1b masks the NLS and Mitf is retained in the cytoplasm. The ratio of M-CSF/Mitf determines the distribution of Mitf between the cytoplasmic and nuclear compartments. As mentioned earlier, both M-CSF and RANKL signaling pathways promote Mitf nuclear accumulation and phosphorylate Mitf (Mansky et al. 2002; Weilbaecher et al. 


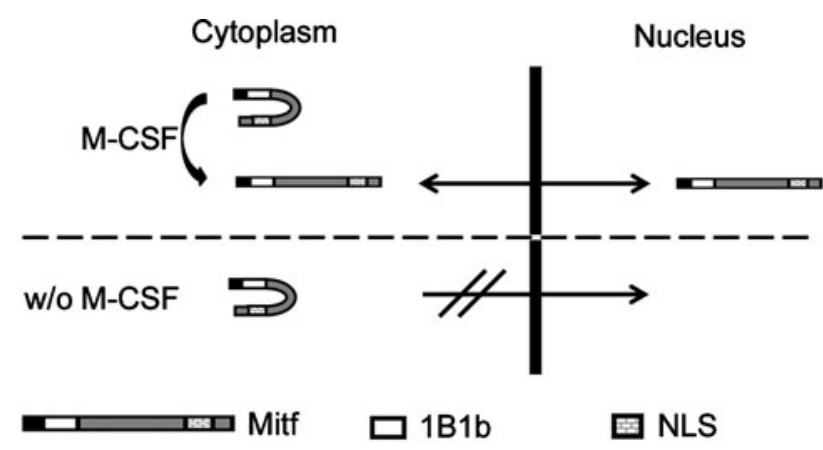

Fig. 7 The proposed NLS masking model for Mitf subcellular localization. $w / o$ without

2001). Future experiments are needed to address whether interference of M-CSF and RANKL phosphorylation sites, serine 73 and serine 307, leads to disruption of the intramolecular NLS masking mechanism. Interestingly, Bronisz et al. demonstrated that dephosphorylation on serine 173 of Mitf promotes Mitf nuclear localization (Bronisz et al. 2006).

Regulation of subcellular localization of Mitf by M-CSF is not an all-or-none response; it is associated with the ratio of M-CSF/Mitf. The domain 1B1b and cell attachment also play roles. Therefore, subcellular localization of Mitf is regulated by different mechanisms that work coordinately in response to various external and internal signals. These coordinated responses ensure precise control of cell's function in the in vivo environment.

Acknowledgments We thank Dr. David E. Fisher for the $\alpha$-Mitf Ab (C5). This study was supported by National Institute of Dental and Craniofacial Research grant R03 DE019490 to YLL.

Open Access This article is distributed under the terms of the Creative Commons Attribution Noncommercial License which permits any noncommercial use, distribution, and reproduction in any medium, provided the original author(s) and source are credited.

\section{References}

Amae S, Fuse N, Yasumoto K, Sato S, Yajima I, Yamamoto H, Udono T, Durlu YK, Tamai M, Takahashi K, Shibahara S (1998) Identification of a novel isoform of microphthalmia-associated transcription factor that is enriched in retinal pigment epithelium. Biochem Biophys Res Commun 247:710-715

Bronisz A, Sharma SM, Hu R, Godlewski J, Tzivion G, Mansky KC, Ostrowski MC (2006) Microphthalmia-associated transcription factor Interactions with 14-3-3 modulate differentiation of committed myeloid precursors. Mol Biol Cell 17:3897-3906

Chitu V, Stanley ER (2006) Colony-stimulating factor-1 in immunity and inflammation. Curr Opin Immunol 18:39-48

Fowles LF, Stacey KJ, Marks D, Hamilton JA, Hume DA (2000) Regulation of urokinase plasminogen activator gene transcription in the RAW264 murine macrophage cell line by macrophage colony-stimulating factor (CSF-1) is dependent upon the level of cell-surface receptor. Biochem J 347(Pt 1):313-320
Fuse N, Yasumoto K, Takeda K, Amae S, Yoshizawa M, Udono T, Takahashi K, Tamai M, Tomita Y, Tachibana M, Shibahara S (1999) Molecular cloning of cDNA encoding a novel microphthalmiaassociated transcription factor isoform with a distinct amino-terminus. J Biochem 126:1043-1051

Granter SR, Weilbaecher KN, Quigley C, Fisher DE (2002) Role for microphthalmia transcription factor in the diagnosis of metastatic malignant melanoma. Appl Immunohistochem Mol Morphol 10:47-51

Hemesath TJ, Steingrimsson E, McGill G, Hansen MJ, Vaught J, Hodgkinson CA, Arnheiter H, Copeland NG, Jenkins NA, Fisher DE (1994) Microphthalmia, a critical factor in melanocyte development, defines a discrete transcription factor family. Genes Dev 8:2770-2780

Hershey CL, Fisher DE (2005) Genomic analysis of the microphthalmia locus and identification of the MITF-J/Mitf-J isoform. Gene 347:73-82

Hogan PG, Chen L, Nardone J, Rao A (2003) Transcriptional regulation by calcium, calcineurin, and NFAT. Genes Dev 17:22052232

Lacey DL, Timms E, Tan HL, Kelley MJ, Dunstan CR, Burgess T, Elliott R, Colombero A, Elliott G, Scully S, Hsu H, Sullivan J, Hawkins N, Davy E, Capparelli C, Eli A, Qian YX, Kaufman S, Sarosi I, Shalhoub V, Senaldi G, Guo J, Delaney J, Boyle WJ (1998) Osteoprotegerin ligand is a cytokine that regulates osteoclast differentiation and activation. Cell 93:165-176

Lagasse E, Weissman IL (1997) Enforced expression of Bcl-2 in monocytes rescues macrophages and partially reverses osteopetrosis in op/op mice. Cell 89:1021-1031

Mansky KC, Sankar U, Han J, Ostrowski MC (2002) Microphthalmia transcription factor is a target of the p38 MAPK pathway in response to receptor activator of NF-kappa B ligand signaling. J Biol Chem 277:11077-11083

Mercurio F, Manning AM (1999) Multiple signals converging on NFkappaB. Curr Opin Cell Biol 11:226-232

Oboki K, Morii E, Kataoka TR, Jippo T, Kitamura Y (2002) Isoforms of mi transcription factor preferentially expressed in cultured mast cells of mice. Biochem Biophys Res Commun 290:12501254

Rohan PJ, Stechschulte DJ, Li Y, Dileepan KN (1997) Macrophage function in mice with a mutation at the microphthalmia (mi) locus. Proc Soc Exp Biol Med 215:269-274

Roundy K, Kollhoff A, Eichwald EJ, Weis JJ, Weis JH (1999) Microphthalmic mice display a B cell deficiency similar to that seen for mast and NK cells. J Immunol 163:6671-6678

Shiohara M, Shigemura T, Suzuki T, Tanaka M, Morii E, Ohtsu H, Shibahara S, Koike K (2009) MITF-CM, a newly identified isoform of microphthalmia-associated transcription factor, is expressed in cultured mast cells. Int J Lab Hematol 31(2):215-226

Stechschulte DJ, Sharma R, Dileepan KN, Simpson KM, Aggarwal N, Clancy J Jr, Jilka RL (1987) Effect of the mi allele on mast cells, basophils, natural killer cells, and osteoclasts in C57Bl/6 J mice. J Cell Physiol 132:565-570

Steingrimsson E, Moore KJ, Lamoreux ML, Ferre-D'Amare AR, Burley SK, Zimring DC, Skow LC, Hodgkinson CA, Arnheiter H, Copeland NG et al (1994) Molecular basis of mouse microphthalmia (mi) mutations helps explain their developmental and phenotypic consequences. Nat Genet 8:256-263

Steingrimsson E, Copeland NG, Jenkins NA (2004) Melanocytes and the microphthalmia transcription factor network. Annu Rev Genet 38:365-411

Takeda K, Yasumoto K, Kawaguchi N, Udono T, Watanabe K, Saito H, Takahashi K, Noda M, Shibahara S (2002) Mitf-D, a newly identified isoform, expressed in the retinal pigment epithelium and monocyte-lineage cells affected by Mitf mutations. Biochim Biophys Acta 1574:15-23 
Takemoto CM, Yoon YJ, Fisher DE (2002) The identification and functional characterization of a novel mast cell isoform of the microphthalmia-associated transcription factor. J Biol Chem 277:30244-30252

Thesingh CW, Scherft JP (1985) Fusion disability of embryonic osteoclast precursor cells and macrophages in the microphthalmic osteopetrotic mouse. Bone 6:43-52

Udono T, Yasumoto K, Takeda K, Amae S, Watanabe K, Saito H, Fuse N, Tachibana M, Takahashi K, Tamai M, Shibahara S (2000)
Structural organization of the human microphthalmia-associated transcription factor gene containing four alternative promoters. Biochim Biophys Acta 1491:205-219

Weilbaecher KN, Motyckova G, Huber WE, Takemoto CM, Hemesath TJ, Xu Y, Hershey CL, Dowland NR, Wells AG, Fisher DE (2001) Linkage of M-CSF signaling to Mitf, TFE3, and the osteoclast defect in Mitf (mi/mi) mice. Mol Cell 8:749-758 